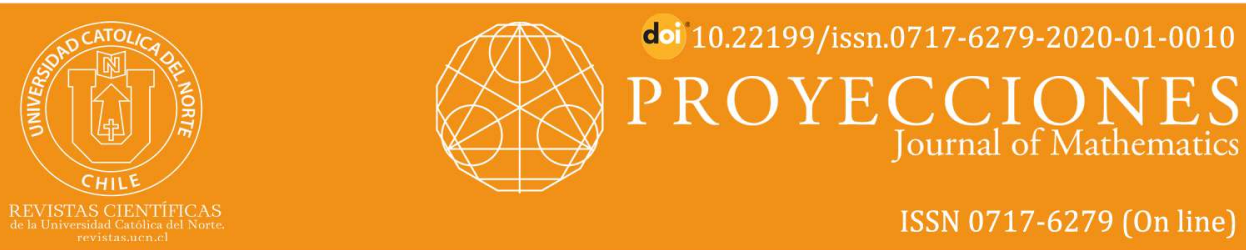

\title{
Some refinements to Hölder's inequality and applications
}

Mohamed Akkouchi ${ }^{1}$

Mohamed Amine Ighachane ${ }^{2}$ (1) orcid.org/0000-0002-4089-5617

Cadi Ayyad University, Dept. of Mathematics, Marrakech, Morocco.

1@akkm555@yahoo.fr ; 2₫ mohamedamineighachane@gmail.com

Received: January2019 | Accepted: May 2019

\section{Abstract:}

We establish some new refinements to the Hölder inequality. We then apply them to provide some refinements to the extended Euler's gamma and beta functions. As another application of our results, we give a new proof of the equivalence between the Hölder inequality and the Cauchy-Schwarz inequality.

Keywords: Inequalities; Young's inequality; Cauchy-Schwarz inequality; Inequalities for extended Beta and Gamma functions.

MSC (2010): 26D15; 33B15; 33B99.

\section{Cite this article as (IEEE citation style):}

M. Akkouchi and M. A. Ighachane, "Some refinements to

Hölder's inequality and applications", Proyecciones (Antofagasta, On line), vol. 39, no. 1, pp. 153-166, Feb. 2020, doi: 10.22199/issn.0717-6279-2020-01-0010. [Accessed dd-mm-yyyy].

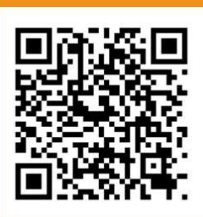

Article copyright: (C) 2020 Mohamed Akkouchi and Mohamed Amine Ighachane. This is an open access article distributed under the terms of the Creative Commons Licence, which permits unrestricted use and distribution provided the original author and source are credited. 


\section{Introduction}

Let $(\Omega, \mathcal{F}, \mu)$ be a measure space ( $\mu$ is a positive measure). For any mesurable functions $f, g: \Omega \mapsto \mathbf{C}$ on $\Omega$, we recall the Hölder's inequality:

$$
\int_{\Omega}|f g| d \mu \leq\left(\int_{\Omega}|f|^{p} d \mu\right)^{\frac{1}{p}}\left(\int_{\Omega}|f|^{q} d \mu\right)^{\frac{1}{q}}, \forall p, q \geq 1 \text { with } \frac{1}{p}+\frac{1}{q}=1 .
$$

If $p=q=2$ then we obtain the cauchy-Schwarz inequality:

$$
\int_{\Omega}|f g| d \mu \leq\left(\int_{\Omega}|f|^{2} d \mu\right)^{\frac{1}{2}}\left(\int_{\Omega}|f|^{2} d \mu\right)^{\frac{1}{2}} .
$$

Their discrete versions are respectively, given by:

$$
\sum_{i=1}^{n}\left|x_{i} y_{i}\right| \leq\left[\sum_{i=1}^{n}\left|x_{i}\right|^{p}\right]^{\frac{1}{p}}\left[\sum_{i=1}^{n}\left|y_{i}\right|^{q}\right]^{\frac{1}{q}}:=\|x\|_{p}\|y\|_{q}, \quad(H)_{d}
$$

and

$$
\sum_{i=1}^{n}\left|x_{i} y_{i}\right| \leq\left[\sum_{i=1}^{n}\left|x_{i}\right|^{2}\right]^{\frac{1}{2}}\left[\sum_{i=1}^{n}\left|y_{i}\right|^{2}\right]^{\frac{1}{2}}:=\|x\|_{2}\|y\|_{2} \quad(C . S)_{d}
$$

which are valid for all positive integer $n$ and all vectors $\left(x_{1}, \ldots, x_{n}\right),\left(y_{1}, \ldots, y_{n}\right) \in \mathbf{K}^{n}$, where the field $\mathbf{K}$ is real or complex.

Easily, we have $(H) \Longrightarrow(C . S)$.

It is natural to raise the question: is the converse true ?.

In fact, the converse was known to be true in the literature (see for instance, [10], [12], [13], [11] and [9]).

There are many connections between classical discrete inequalities. Some of these connections were noted in several chapters of the book [13], where in particular the equivalence $(H)_{d} \Longleftrightarrow(C . S)_{d}$ was obtained after some intermidiate results.

It is known, from the book [12] of A. W. Marshall and I. Olkin, that the Cauchy-Schwarz inequality implies Lyapunov's inequality which itself implies the arithmetic-geometric mean inequality. We can say that, in a sense, the arithmetic-geometric mean inequality, Holder's inequality, the Cauchy-Schwarz inequality, and Lyapunov's inequality are all equivalent [[12], p. 457]. 
This paper is organised as follows: In section 2, we give some refinements to Young's inequality. In section 3, we provide some new refinements of Hölder's inequality. In section 4, we give applications to the extended gamma function. In section 5, we give applications to the extended beta function. In section 6 , we apply our results to etablish the equivalence between the integral inequalities $(H)$ and $(C-S)$. In section 7 , we present some conclusions and discuss the possibility of other applications, of our results, to provide new refinements to certain classical inequalities involving various special functions.

We notice that [15] contains a good list of references on the extended Euler's gamma and beta functions.

We point out that the inequalities obtained in the sections 4 and 5 generalize and refine some well known results concerning the gamma and beta functions and their extensions (see [13], [8], [7], and [15]).

\section{Refinements of Young's inequality}

Let $a, b$ be two positive numbers and let $\alpha \in[0,1]$. We denote by $Y(\alpha)$ the Young's inequality:

$$
a^{\alpha} b^{1-\alpha} \leq \alpha a+(1-\alpha) b
$$

If $\alpha=1 / 2$ then we find the Cauchy-Jensen (or geometric mean -arithmetic mean) inequality :

$$
\sqrt{a b} \leq \frac{a+b}{2}
$$

To prove our first result we need to recall the following result which is a consequence from the paper [1].

Theorem $2.1([1])$. Let $\left(X_{n}\right)_{n=1}^{\infty}$ be a sequence of real integrable r.v.s which are independent and equidistributed defined on a Probability space $(\Omega, \mathcal{F}, P)$ and taking values in an interval $I$ of $\mathbf{R}$. Let $\phi: I \rightarrow \mathbf{R}$ be a continuous convex function. Then the following inequalities hold:

$\phi\left(E\left[X_{1}\right]\right) \leq E\left[\phi\left(Y_{n+1}\right)\right] \leq E\left[\phi\left(Y_{n}\right)\right] \leq E\left[\phi\left(X_{1}\right)\right]$ for all positive integer $n$,

where $Y_{n}:=\frac{1}{n} \sum_{i=1}^{n} X_{n}$ for all positive integer $n$ and $E[X]$ designates the esperance for all integrable random variable $X$ on $\Omega$.

Concerning Young's inequality, we establish the following result. 
Theorem 2.2. Let $a$ and $b$ two positive numbers. Then for all $n \geq 1$ and $\alpha \in] 0,1[$ we have

$$
a^{\alpha} b^{1-\alpha} \leq\left(\alpha a^{\frac{1}{n}}+(1-\alpha) b^{\frac{1}{n}}\right)^{n} \leq \alpha a+(1-\alpha) b,
$$

moreover if we set $U_{n}(\alpha, a, b):=\left(\alpha a^{\frac{1}{n}}+(1-\alpha) b^{\frac{1}{n}}\right)^{n}$ then $\left(U_{n}(\alpha, a, b)\right)_{n \geq 1}$ is a nonincreasing sequence satisfying

$$
\lim _{n \rightarrow \infty} U_{n}(\alpha, a, b)=a^{\alpha} b^{1-\alpha} .
$$

Proof. (a) Choose $x=a^{1 / n}$ et $y=b^{1 / n}$. by Young inequality we have

$$
x^{\alpha} y^{1-\alpha}=a^{\frac{\alpha}{n}} b^{\frac{1-\alpha}{n}} \leq\left(\alpha a^{1 / n}+(1-\alpha) b^{1 / n}\right)
$$

and so

$$
a^{\alpha} b^{1-\alpha} \leq\left(\alpha a^{1 / n}+(1-\alpha) b^{1 / n}\right)^{n} .
$$

By convexity of function $f(t)=t^{n} n \geq 2$ we find that

$$
\left(\alpha a^{1 / n}+(1-\alpha) b^{1 / n}\right)^{n} \leq \alpha a+(1-\alpha) b
$$

It follows that

$$
a^{\alpha} b^{1-\alpha} \leq\left(\alpha a^{\frac{1}{n}}+(1-\alpha) b^{\frac{1}{n}}\right)^{n} \leq \alpha a+(1-\alpha) b .
$$

We have

$$
\begin{aligned}
\lim _{n \rightarrow+\infty} U_{n}(\alpha, a, b) & =\lim _{n \rightarrow+\infty}\left(\alpha a^{1 / n}+(1-\alpha) b^{1 / n}\right)^{n} \\
& =\lim _{n \rightarrow+\infty} \exp \left(n \ln \left(\alpha a^{1 / n}+(1-\alpha) b^{1 / n}\right)\right) \\
& =\lim _{h \rightarrow 0} \exp \left(\frac{1}{h} \ln \left(\alpha a^{h}+(1-\alpha) b^{h}\right)\right) \\
& =\lim _{h \rightarrow 0} \exp \left(\frac{1}{h} \ln \left(b^{h}\left(1+\alpha\left(\left(\frac{a}{b}\right)^{h}-1\right)\right)\right)\right. \\
& =b \lim _{h \rightarrow 0} \exp \left(\frac{1}{h} \ln \left(\left(1+\alpha\left(\left(\frac{a}{b}\right)^{h}-1\right)\right)\right)\right. \\
& =b \exp \alpha \ln \left(\frac{a}{b}\right)=a^{\alpha} b^{1-\alpha}
\end{aligned}
$$

(b) It remains to show that the sequence $\left(U_{n}(\alpha, a, b)\right)$ is nonincreasing. To this end, we apply Theorem 2.1, for independent and equidistributed 
random variables with the same probability law $\mu:=\alpha \delta_{\ln (a)}+(1-\alpha) \delta_{\ln (b)}$, where $\delta_{c}$ means the Dirac measure with mass one concentrated at the point $c$ for each $c \in \mathbf{R}$.

(c) We set $I:=\mathbf{R}$ and consider the convex function $x \mapsto e^{x}$ defined on $I=\mathbf{R}$.

Indeed, we observe that for all positive integer $n$, we have

$$
U_{n}(\alpha, a, b)=E\left(e^{\frac{X_{1}+X_{2}+\ldots X_{n}}{n}}\right)
$$

We use Theorem 2.1 to conclude that this sequence is nondecreasing. This ends the proof.

In the next result, we prove that the inequalities $(Y(\alpha))$ and $\left(Y\left(\frac{1}{2}\right)\right)$ are equivalent.

Theorem 2.3. The inequalities of Young $Y\left(\frac{1}{2}\right)$ and $Y(\alpha)$ are equivalent.

Proof. Suppose that $Y\left(\frac{1}{2}\right)$ is statisfied, then for all $k$ a postive integer, we have

$$
\left(a_{1} \ldots a_{2^{n}}\right)^{1 / 2^{n}} \leq \frac{1}{2^{n}}\left(a_{1}+\ldots .+a_{2^{n}}\right) .
$$

We choose $a_{i}=a$ for $1 \leq i \leq k$ and $a_{i}=b$ for $k+1 \leq i \leq 2^{n}$. Then we get

$$
\left(a^{k} b^{2^{n}-k}\right)^{1 / 2^{n}} \leq 1 / 2^{n}\left(k a+\left(2^{n}-k\right) b\right)
$$

It follows that

$$
a^{\frac{k}{2^{n}}} b^{1-\frac{k}{2^{n}}} \leq \frac{k}{2^{n}} a+\left(1-\frac{k}{2^{n}}\right) b
$$

By density of the set $\left\{\frac{k}{2^{n}}: 1 \leq k \leq 2^{n}\right\}$ in $[0,1]$ we obtain

$$
a^{\alpha} b^{1-\alpha} \leq \alpha a+(1-\alpha) b
$$

This completes the proof.

We point out that J. M. Aldaz (see [3] and the references therein) discussed other types of improvements to the AM-GM inequality and gave some applications of them. 


\section{Refinements of Hölder's inequality}

The main result of this section reads as follows.

Theorem 3.1. Let $p$ and $q$ tow numbers such that $1 / p+1 / q=1$ and $p, q>1$, and $f, g$ nonnegative $\mu$-mesurable fonctions. Then for all $n \geq 2$ we have:

$$
\begin{aligned}
\int_{\Omega}|f(x) g(x)| d \mu(x) & \leq\left(\frac{1}{p^{n}}+\frac{1}{q^{n}}\right)\|f\|_{p}\|g\|_{q} \\
& +\sum_{k=1}^{n-1}\left(\begin{array}{l}
n \\
k
\end{array}\right) \frac{1}{p^{k} q^{n-k}}\|f\|_{p}^{1-\frac{k p}{n}}|| g \|_{q}^{1-\frac{(n-k) q}{n}} \int_{\Omega}|f|^{\frac{k p}{n}}|g|^{\frac{(n-k) q}{n}} d \mu \\
& \leq\left(\int_{\Omega}|f|^{p} d \mu\right)^{\frac{1}{p}}\left(\int_{\Omega}|g|^{q} d \mu\right)^{\frac{1}{q}},
\end{aligned}
$$

where $\left(\begin{array}{l}n \\ k\end{array}\right):=\frac{n !}{(n-k) ! k !}$ is the usual binomial coefficient, for all $k \in\{0,1,2, \ldots n\}$.

Proof. we have

$$
a b=\left(a^{p}\right)^{\frac{1}{p}}\left(b^{q}\right)^{\frac{1}{q}} \leq\left(\frac{1}{p} a^{\frac{p}{n}}+\frac{1}{q} b^{\frac{q}{n}}\right)^{n}=\sum_{k=0}^{n}\left(\begin{array}{l}
n \\
k
\end{array}\right)\left(\frac{a^{p / n}}{p}\right)^{k}\left(\frac{b^{q / n}}{q}\right)^{n-k} .
$$

We set $a=\frac{|f(x)|}{\|f\|_{p}}$ and $b=\frac{|g(x)|}{\|g\|_{q}}$ then

$\frac{|f(x) g(x)|}{\|f\|_{p}\|g\|_{q}} \leq \frac{|f(x)|^{p}}{p^{n}\|f\|_{p}^{p}}+\frac{|g(x)|^{q}}{q^{n}\|g\|_{q}^{q}}+\sum_{k=1}^{n-1}\left(\begin{array}{l}n \\ k\end{array}\right) \frac{1}{p^{k} q^{n-k}} \frac{|f(x)|^{(k p) / n}}{\|f\|_{p}^{(k p) / n}} \frac{|g(x)|^{(n-k) q / n}}{\|g\|_{q}^{(n-k) q / n}}$.

It follows that

$$
\int_{\Omega} \frac{|f(x) g(x)|}{\|f\|_{p}|| g \|_{q}} d \mu(x) \leq \frac{1}{p^{n}}+\frac{1}{q^{n}}+\int_{\Omega}\left(\sum_{k=1}^{n-1}\left(\begin{array}{l}
n \\
k
\end{array}\right) \frac{1}{p^{k} q^{n-k}} \frac{|f(x)|^{(k p) / n}}{\|f\|_{p}^{(k p) / n}} \frac{|g(x)|^{(n-k) q / n}}{\|g\|_{q}^{(n-k) q / n}}\right) d \mu(x) .
$$

Therefore, we have

$$
\begin{aligned}
\int_{\Omega}|f g| d \mu & \leq\left(\frac{1}{p^{n}}+\frac{1}{q^{n}}\right)\|f\|_{p}\|g\|_{q} \\
& +\sum_{k=1}^{n-1}\left(\begin{array}{l}
n \\
k
\end{array}\right) \frac{1}{p^{k} q^{n-k}}\|f\|_{p}^{1-\frac{k p}{n}}\|g\|_{q}^{1-\frac{(n-k) q}{n}} \int_{\Omega}|f|^{(k p) / n}|g|^{(n-k) q / n} d \mu .
\end{aligned}
$$

This proves the first inequality in the right hand side of (3.1). 
For the second inequality in the right hand side of (3.1), we make use of Hölder's inequality. For $1 \leq k \leq n-1$, We get

$\int_{\Omega}|f|^{\frac{k p}{n}}|g|^{\frac{(n-k) q}{n}} d \mu \leq\left[\int_{\Omega}\left(|f|^{\frac{k p}{n}}\right)^{\frac{n}{k}} d \mu\right]^{\frac{k}{n}}\left[\int_{\Omega}\left(|g|^{\frac{(n-k) q}{n}}\right)^{\frac{n}{n-k}} d \mu\right]^{\frac{n-k}{n}}=\|f\|_{p^{\frac{k p}{n}}}^{\|g\|^{\frac{(n-k) q}{n}} .}$

Taking into account of these inequalities, we deduce the last inequality. This ends the proof. For the discrete case, we have the following inequality:

Corollary 3.1. Let $p$ and $q$ two positive numbers show that $p \geq 1$ and $\frac{1}{p}+\frac{1}{q}=1$. For all vectors $\left(x_{1}, \ldots, x_{n}\right),\left(y_{1}, \ldots, y_{n}\right) \in \mathbf{K}^{n}$ with $n \geq 1$, we have:

$$
\begin{aligned}
\sum_{i=1}^{m}\left|x_{i} y_{i}\right| & \leq\left(\frac{1}{p^{n}}+\frac{1}{q^{n}}\right)\|x\|_{p}\|y\|_{q} \\
& +\sum_{k=1}^{n-1}\left(\begin{array}{l}
n \\
k
\end{array}\right) \frac{1}{p^{k} q^{n-k}}\|x\|_{p}^{1-\frac{k p}{n}}\|y\|_{q}^{1-\frac{(n-k) q}{n}} \sum_{i=1}^{m}\left|x_{i}\right|^{\frac{k p}{n}}\left|y_{i}\right|^{\frac{(n-k) q}{n}} \\
& \leq\|x\|_{p}\|y\|_{q}
\end{aligned}
$$

\section{Applications to the extended gamma function}

In $[8]$ we find a proof of the following result.

Theorem 4.1. ([8]) Let $a, b$ be nonnegative numbers with $a+b=1$ and $x, y$ be positive numbers. Then

$$
\Gamma(a x+b y) \leq \Gamma(x)^{a} \Gamma(y)^{b},
$$

i.e., the mapping $\Gamma$ is logarithmically convex on $(0,+\infty)$.

This result was extended to the so called extended Gamma functions.

In 1994, Chaudhry and Zubair (see [7]) introduced the extended gamma function by setting

$$
\Gamma_{w}(x):=\int_{0}^{+\infty} t^{x-1} e^{-t-w t^{-1}} d t, \quad \Re(x)>0, \quad w \in[0,+\infty) .
$$

If $w=0$, then $\Gamma_{0}$ coincides with the classical gamma function usually denoted by $\Gamma$.

The generalization of the inequality (4.1) to the setting was made in [7] and [15]. 
Theorem 4.2. ([7] - [15]) Let $w \geq 0$. Let $a, b$ be nonnegative numbers with $a+b=1$ and $x, y$ be positive numbers. Then

$$
\Gamma_{w}(a x+b y) \leq \Gamma_{w}(x)^{a} \Gamma_{w}(y)^{b},
$$

i.e., the mapping $\Gamma_{w}$ is logarithmically convex on $(0,+\infty)$.

By using Theorem 3.1, we obtain the following refinements of the inequality (4.2) for the extended gamma function.

Theorem 4.3. Let $w \geq 0$. Let $a, b \geq 0$ with $a+b=1$ and $x, y>0$. Then for all $n \geq 2$

$$
\begin{aligned}
\Gamma_{w}(a x+b y) & \leq\left(a^{n}+b^{n}\right) \Gamma_{w}(x)^{a} \Gamma_{w}(y)^{b} \\
& +\sum_{k=1}^{n-1}\left(\begin{array}{l}
n \\
k
\end{array}\right) a^{k} b^{n-k} \Gamma_{w}(x)^{a-\frac{k}{n}} \Gamma_{w}(y)^{b-\frac{n-k}{n}} \Gamma_{w}\left(\frac{k}{n} x+\frac{n-k}{n} y\right) \\
& \leq \Gamma_{w}(x)^{a} \Gamma_{w}(y)^{b} .
\end{aligned}
$$

Proof. To apply our Theorem 3.1 , we set $\Omega:=(0+\infty)$ and take the measure $\mu$ given by $d \mu(t):=e^{-t-w t^{-1}} d t$. Then we choose $f(s)=s^{a(x-1)}$ and $g(s)=s^{b(y-1)}$ and $p=1 / a, b=1 / b$. Then we have $1 / p+1 / q=a+b=1$ and easy computations give the following:

$\|f\|_{p}=\left(\int_{0}^{\infty} s^{(x-1)} d \mu(s)\right)^{a}=\Gamma_{w}(x)^{a}, \quad\|g\|_{q}=\left(\int_{0}^{\infty} s^{(y-1)} d \mu(s)\right)^{b}=\Gamma_{w}(y)^{b}$

and

$$
\int_{0}^{\infty} s^{a(x-1)} s^{b(y-1)} d \mu(s)=\Gamma_{w}(a x+b y) .
$$

Then by Theorem 3.1, we have

$$
\begin{aligned}
& \Gamma_{w}(a x+b y) \leq\left(a^{n}+b^{n}\right) \Gamma_{w}(x)^{a} \Gamma_{w}(y)^{b} \\
& +\sum_{k=1}^{n-1}\left(\begin{array}{l}
n \\
k
\end{array}\right) a^{k} b^{n-k} \Gamma_{w}(x)^{a-\frac{k}{n}} \Gamma_{w}(y)^{b-\frac{n-k}{n}} \int_{0}^{\infty} s^{\frac{k}{n}(x-1)} s^{\frac{(n-k)}{n}(y-1)} d \mu(s) \\
& =\left(a^{n}+b^{n}\right) \Gamma_{w}(x)^{a} \Gamma_{w}(y)^{b} \\
& +\sum_{k=1}^{n-1}\left(\begin{array}{l}
n \\
k
\end{array}\right) a^{k} b^{n-k} \Gamma_{w}(x)^{a-\frac{k}{n}} \Gamma_{w}(y)^{b-\frac{n-k}{n}} \Gamma_{w}\left(\frac{k}{n} x+\frac{n-k}{n} y\right) .
\end{aligned}
$$

By using the inequality (4.2), for all $1 \leq k \leq n-1$, we have

$$
\Gamma_{w}\left(\frac{k}{n} x+\frac{n-k}{n} y\right) \leq \Gamma_{w}(x)^{\frac{k}{n}} \Gamma_{w}(y)^{\frac{n-k}{n}} .
$$


Therefore, we have

$$
\begin{aligned}
\Gamma_{w}(a x+b y) & \leq\left(a^{n}+b^{n}\right) \Gamma_{w}(x)^{a} \Gamma_{w}(y)^{b} \\
& +\sum_{k=1}^{n-1}\left(\begin{array}{l}
n \\
k
\end{array}\right) a^{k} b^{n-k} \Gamma_{w}(x)^{a-\frac{k}{n}} \Gamma_{w}(y)^{b-\frac{n-k}{n}} \Gamma_{w}\left(\frac{k}{n} x+\frac{n-k}{n} y\right) \\
& \leq \Gamma_{w}(x)^{a} \Gamma_{w}(y)^{b} .
\end{aligned}
$$

This ends the proof.

\section{Applications to the extended beta function}

S. S. Dragomir et al. [8] proved the following result.

Theorem 5.1. ([8]) Let $a, b$ be nonnegative numbers with $a+b=1$. Then, for all $\left(p_{1}, q_{1}\right),\left(p_{2}, q_{2}\right) \in(0,+\infty)^{2}$ we have

$$
B\left(a p_{1}+b q_{1}, a p_{2}+b q_{2}\right) \leq B\left(p_{1}, p_{2}\right)^{a} B\left(q_{1}, q_{2}\right)^{b},
$$

i.e., the mapping $B$ is logarithmically convex on $(0,+\infty)^{2}$ as a function of two variables.

In 1997, Chaudhry et al. (see [6]) introduced the extended Euler's beta function by setting

$B_{w}(x, y):=\int_{0}^{1} t^{x-1}(1-t)^{y-1} e^{-w / t(1-t)} d t, \quad \Re(x), \Re(y)>0, \quad w \in[0,+\infty)$.

If $w=0$, then $\beta_{0}$ coincides with the classical beta function usually denoted by $\beta$.

The generalization of the inequality (5.1) to the setting was made in [6] and $[15]$.

Theorem 5.2. ([6] - [15]) Let $w \geq 0$. Let $a, b$ be nonnegative numbers with $a+b=1$. Then, for all $\left(p_{1}, q_{1}\right),\left(p_{2}, q_{2}\right) \in(0,+\infty)^{2}$ we have

$$
B_{w}\left(a p_{1}+b q_{1}, a p_{2}+b q_{2}\right) \leq B_{w}\left(p_{1}, p_{2}\right)^{a} B_{w}\left(q_{1}, q_{2}\right)^{b},
$$

i.e., the mapping $B_{w}$ is logarithmically convex on $(0,+\infty)^{2}$ as a function of two variables.

By using Theorem 3.1, we obtain the following refinements of the inequality (5.2). 
Theorem 5.3. Let $w \geq 0$. Let $a, b \geq 0$ with $a+b=1$ and $x, y \geq 0$. Then for all $n \geq 2$, we have

$$
\begin{aligned}
& B_{w}\left(a p_{1}+b q_{1}, a p_{2}+b q_{2}\right) \leq\left(a^{n}+b^{n}\right) B_{w}\left(p_{1}, p_{2}\right)^{a} B_{w}\left(q_{1}, q_{2}\right)^{b} \\
&+\sum_{k=1}^{n-1}\left(\begin{array}{l}
n \\
k
\end{array}\right) a^{k} b^{n-k} B_{w}\left(p_{1}, p_{2}\right)^{a-\frac{k}{n}} B_{w}\left(q_{1}, q_{2}\right)^{b-\frac{n-k}{n}} B_{w}\left(\frac{k}{n}\left(p_{1}, p_{2}\right)+\frac{n-k}{n}\left(q_{1}, q_{2}\right)\right) \\
& \leq B_{w}\left(p_{1}, p_{2}\right)^{a} B_{w}\left(q_{1}, q_{2}\right)^{b}
\end{aligned}
$$

Proof. Let $\left.\left(p_{1}, p_{2}\right),\left(q_{1}, q_{2}\right) \in\right] 0,+\infty\left[\left[^{2}\right.\right.$ and $a, b \geq 0$ with $a+b=1$. We have

$$
\begin{aligned}
B_{w}\left(a p_{1}+b q_{1}, a p_{2}+b q_{2}\right) & =\int_{0}^{1} t^{a p_{1}+b q_{1}-1}(1-t)^{a p_{2}+b q_{2}-1} e^{-w / t(1-t)} d t \\
& =\int_{0}^{1}\left[t^{p_{1}-1}(1-t)^{p_{2}-1}\right]^{a}\left[t^{q_{1}-1}(1-t)^{q_{2}-1}\right]^{b} e^{-w / t(1-t)} d t .
\end{aligned}
$$

We apply Theorem 3.1, by setting $\Omega:=(0,1)$ and considering the measure $d \mu(t):=e^{-w / t(1-t)} d t$ on $(0,1)$. We set $p=1 / a, q=1 / b$.

we have:

Choose $f(t)=\left[t^{p_{1}-1}(1-t)^{p_{2}-1}\right]^{a}$ and $g(t)=\left[t^{q_{1}-1}(1-t)^{q_{2}-1}\right]^{b}$. Then

$$
\|f\|_{p}=\left(\int_{0}^{1}\left[t^{p_{1}-1}(1-t)^{p_{2}-1}\right] d \mu(t)\right)^{a}=B_{w}\left(p_{1}, p_{2}\right)^{a}
$$

and

$$
\|g\|_{q}=\left(\int_{0}^{1}\left[t^{q_{1}-1}(1-t)^{q_{2}-1}\right] d \mu(t)\right)^{b}=B_{w}\left(q_{1}, q_{2}\right)^{b} .
$$

Then

$$
\begin{aligned}
\int_{0}^{1}|f(t)|^{\frac{k}{a n}}|g(t)|^{\frac{n-k}{b n}} d \mu(t) & =\int_{0}^{1}\left[t^{p_{1}-1}(1-t)^{p_{2}-1}\right]^{a\left(\frac{k}{a n}\right)}\left[t^{q_{1}-1}(1-t)^{q_{2}-1}\right]^{b \frac{(n-k)}{b n}} d \mu(t) \\
& =\int_{0}^{1}\left[t^{p_{1}-1}(1-t)^{p_{2}-1}\right]^{\frac{k}{n}}\left[t^{q_{1}-1}(1-t)^{q_{2}-1}\right]^{\frac{(n-k)}{n}} d \mu(t) \\
& =\int_{0}^{1}\left[t^{\frac{k}{n}\left(p_{1}-1\right)}(1-t)^{\frac{n-k}{n}\left(q_{2}-1\right)}\right]\left[t^{\frac{n-k}{n}\left(q_{1}-1\right)}(1-t)^{\frac{k}{n}\left(p_{2}-1\right)}\right] d \mu(t)
\end{aligned}
$$




$$
\begin{aligned}
& =\int_{0}^{1}\left[t^{\frac{k}{n}\left(p_{1}-1\right)+\frac{n-k}{n}\left(q_{1}-1\right)}(1-t)^{\frac{k}{n}\left(p_{2}-1\right)+\frac{n-k}{n}\left(q_{2}-1\right)}\right] d \mu(t) \\
& =\int_{0}^{1}\left[t^{\frac{k}{n} p_{1}+\frac{n-k}{n} q_{1}-1}(1-t)^{\frac{k}{n} p_{2}+\frac{n-k}{n} q_{2}-1}\right] d \mu(t) \\
& =B_{w}\left(\frac{k}{n}\left(p_{1}, p_{2}\right), \frac{n-k}{n}\left(q_{1}, q_{2}\right)\right) .
\end{aligned}
$$

By virtue of Theorem 3.1, we have

$$
\begin{gathered}
B_{w}\left(a\left(p_{1}, p_{2}\right)+b\left(q_{1}, q_{2}\right)\right) \leq\left(a^{n}+b^{n}\right) B_{w}\left(p_{1}, p_{2}\right)^{a} B_{w}\left(q_{1}, q_{2}\right)^{b} \\
+\sum_{k=1}^{n-1}\left(\begin{array}{l}
n \\
k
\end{array}\right) a^{k} b^{n-k} B_{w}\left(p_{1}, p_{2}\right)^{a-\frac{k}{n}} B_{w}\left(q_{1}, q_{2}\right)^{b-\frac{n-k}{n}} B_{w}\left(\frac{k}{n}\left(p_{1}, q_{2}\right)+\frac{n-k}{n}\left(q_{1}, q_{2}\right)\right) .
\end{gathered}
$$

By the inequality (5.2), we have

$$
B_{w}\left(\frac{k}{n}\left(p_{1}, p_{2}\right), \frac{n-k}{n}\left(q_{1}, q_{2}\right)\right) \leq B_{w}\left(\left(p_{1}, p_{2}\right)\right)^{\frac{k}{n}} B_{w}\left(\left(q_{1}, q_{2}\right)\right)^{\frac{n-k}{n}} .
$$

Therefore

$$
\begin{gathered}
B_{w}\left(a\left(p_{1}, q_{1}\right)+b\left(p_{2}, q_{2}\right)\right) \leq\left(a^{n}+b^{n}\right) B_{w}\left(p_{1}, p_{2}\right)^{a} B_{w}\left(q_{1}, q_{2}\right)^{b} \\
+\sum_{k=1}^{n-1}\left(\begin{array}{c}
n \\
k
\end{array}\right) a^{k} b^{n-k} B_{w}\left(p_{1}, p_{2}\right)^{a-\frac{k}{n}} B_{w}\left(q_{1}, q_{2}\right)^{b-\frac{n-k}{n}} B_{w}\left(\frac{k}{n}\left(p_{1}, q_{1}\right)+\frac{n-k}{n}\left(p_{2}, q_{2}\right)\right) \\
\leq B_{w}\left(p_{1}, p_{2}\right)^{a} B_{w}\left(q_{1}, q_{2}\right)^{b} .
\end{gathered}
$$

This ends the proof.

\section{Equivalence of Hölder's and Cauchy-Schwarz inequalities}

In 2006, Y-C Li and S-Y Shaw [11] gave a proof of Hölder's inequality by using the Cauchy-Schwarz inequality. Their method lies on the fact that the convexity of a function on an open and finite interval is equivalent to continuity and midconvexity.

In 2007, the equivalence between the integral inequalities $(H)$ and $(C-$ $S$ ) was studied by C. Finol and M. Wójtowicz in [9]. They gave a proof that $(C-S)$ implies $(H)$ by using density arguments and mathematical induction. in $[2]$.

A discussion of the implication $(C-S) \Rightarrow(H)$ was recently reported 
The aim of this section is to give a proof of this implication by using Theorem 3.1. By this way, we provide a new proof of the equivalence between the Hölder's and Cauchy-Schwarz inequalities.

Theorem 6.1. Let $p$ and $q$ two numbers show that $p>1$ and $\frac{1}{p}+\frac{1}{q}=1$. Then

$$
\begin{aligned}
\int_{\Omega}|f(x) g(x)| d \mu(x) & \leq\left(1-\frac{2}{p q}\right)\|f\|_{p}\|g\|_{q} \\
& +\frac{2}{p q}\|f\|_{p}^{1-\frac{p}{2}}\|g\|_{q}^{1-\frac{q}{2}} \int_{\Omega}|f(x)|^{\frac{p}{2}}|g(x)|^{\frac{q}{2}} d \mu(x) \\
& \leq\left(\int_{\Omega}|f(x)|^{p} d \mu(x)\right)^{\frac{1}{p}}\left(\int_{\Omega}|g(x)|^{q} d \mu(x)\right)^{\frac{1}{q}} .
\end{aligned}
$$

Proof. The first inequality in the right hand side is implied by Theorem 3.1 , for $n=2$.

For the second inequality in the right hand side, by using CauchySchwarz inequality, we get

$$
\left.\int_{\Omega}|f|^{\frac{p}{2}}|g|^{\frac{q}{2}} d \mu \leq\left(\int_{\Omega}\left(|f|^{\frac{p}{2}}\right)^{2}\right)^{1 / 2} \int_{\Omega}\left(|g(x)|^{\frac{q}{2}}\right)^{2}\right)^{1 / 2}=\|f\|_{p}^{p / 2}\|g\|_{q}^{q / 2}
$$

so

$$
\left(1-\frac{2}{p q}\right)\|f\|_{p}\|g\|_{q}+\frac{2}{p q}\|f\|_{p}^{1-\frac{p}{2}}\|g\|_{q}^{1-\frac{q}{2}} \int_{\Omega}|f|^{\frac{p}{2}}|g|^{\frac{q}{2}} d \mu \leq\|f\|_{p}\|g\|_{q} .
$$

Thus our result is proved.

\section{Conclusions}

In this paper, we have given some new refinements to the Hölder inequality. We have applied them to provide some refinements to the extended Euler's gamma and beta functions. By using our main results, we have given a new proof of the equivalence between the Hölder inequality and the Cauchy-Schwarz inequality. We expect in a near future to look for applications to other special functions like the incomplete gamma function, Polygamma functions, Exponential integral function, Abramowitz's function and Hurwitz-Lerch zeta function. We point out some new inequalities for these functions were recently obtained by P. K. Bhandari and S. K. Bissu in [5]. We hope that our results allow some improvements to certain inequalities obtained in [5]. We expect also to apply our results to 
the Struve functions and to their generalizations. We point out that K. S. Nisar et al. studied certain inequalities involving the k-Struve function in [14] and studied the monotonicity properties of the generalized Struve functions in [4]. We expect that our results shoud find some applications to improve some log-convex inequalities established in [14] and [4].

\section{Acknowledgement.}

The authors would like to express their deep thanks to the anonymous referee for his/her comments and suggestions on the initial version of the manuscript which lead to the improvement of this paper.

\section{References}

[1] M. Akkouchi, "Inequalities for real random variables connected with Jensen's inequality and applications", Rivista di matematica della Universitá di Parma, vol. 6, no. 3, pp. 113-125, 2000. [0n line]. Available: https://bit.ly/2vt3mVz

[2] M. Akkouchi, "Cauchy-Schwarz inequality implies Hölder's inequality", RGMIA Research report collection, vol 21, Art. ID. 48, 2018. [On line]. Available: https://bit.ly/2RCwthM

[3] J. M. Aldaz, "Self improvement of the inequality between arithmetic and geometric means", Journal of mathematical inequalities, vol. 3, no. 2, pp. 213-216, 2009, doi: 10.7153/jmi-03-21.

[4] R. M. Ali, S. R. Mondal, and K. S. Nisar, "Monotonicity properties of the generalized Struve functions", Journal of korean mathematical society, vol. 54, no. 2, pp. 575-598, Mar. 2017, doi: 10.4134/JKMS.j160137.

[5] P. K. Bhandari and S. K. Bissu, "Inequalities via Hölder's inequality", Scholars journal of research in mathematics and computer science, vol. 2, no. 2, pp. 124-129, 2018. [On line]. Available: https://bit.ly/2t8TYFT

[6] M. A. Chaudhry, A. Qadir, M. Rafique, and S. M. Zubair, "Extension of Euler's beta function", Journal of computational and applied mathematics, vol. 78, no. 1, pp. 19-32, Feb. 1997, doi: 10.1016/S03770427(96)00102-1.

[7] M. A. Chaudhry and S. M. Zubair, On a class of incomplete gamma functions with applications. Boca Raton, FL: Chapman \& Hall/CRC, 2002. 
[8] S. S. Dragomir, R. P. Agarwal and N. S. Barnett, "Inequalities for beta and gamma functions via some classical and new integral inequalities", Journal of inequalities and applications, vol. 5, no. 2, pp. 103-165, 2000, doi: 10.1155/S1025583400000084.

[9] C. Finol and M. Wojtowicz, "Cauchy-Schwarz and Hölder's inequalities are equivalent", Divulgaciones matemáticas, vol. 15, no. 2, pp. 143-147, 2007. [On line]. Available: https://bit.ly/20bN36h

[10] C. A. Infantozzi, "An introduction to relations among inequalities. The November Meeting in Cleveland, Ohio November 25, 1972", Notices of the American mathematical society, no. 141, pp. A819-A820, 1972. [On line]. Available: https://bit.ly/2U2Y6lY

[11] Y-C. Li and S-Y. Shaw, "A proof of Hölder's inequality using the CauchySchwarz inequality", Journal of inequalities in pure and applied mathematics, vol. 7, no. 2, Art. ID. 62, 2006. [On line]. Available: https://bit.ly/208IYQj

[12] A. W. Marshall and I. Olkin, Inequalities: theory of majorization and its applications. New York, NY: Academic Press, 1979.

[13] D. S. Mtirinovic, J. E. Pecaric and A. M. Fink, Classical and new inequalities in analysis. Dordrecht: Kluwer Academic Publishers, 1993.

[14] K. S. Nisar, S. R. Mondal, and J. Choi, "Certain inequalities involving the k-Struve function", Journal of inequalities and applications, Art. ID. 71, Apr. 2017, doi: 10.1186/s13660-017-1343-x.

[15] K. S. Nisar, F. Qi, G. Rahman, S. Mubeen, and M. Arshad, "Some inequalities involving the extended gamma function and the Kummer confluent hypergeometric k-function", Journal of inequalities and applications, Art. ID. 135, Jun. 2018, doi: 10.1186/s13660-018-1717-8. 